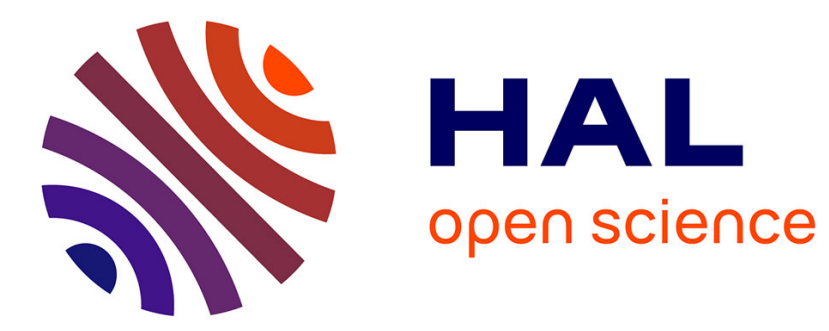

\title{
Human-oriented design of collaborative robots
}

Pauline Maurice, Vincent Padois, Yvan Measson, Philippe Bidaud

\section{To cite this version:}

Pauline Maurice, Vincent Padois, Yvan Measson, Philippe Bidaud. Human-oriented design of collaborative robots. International Journal of Industrial Ergonomics, 2017, 57, pp.88 - 102. 10.1016/j.ergon.2016.11.011 . hal-01428778

\section{HAL Id: hal-01428778 https://hal.science/hal-01428778}

Submitted on 6 Jan 2017

HAL is a multi-disciplinary open access archive for the deposit and dissemination of scientific research documents, whether they are published or not. The documents may come from teaching and research institutions in France or abroad, or from public or private research centers.
L'archive ouverte pluridisciplinaire HAL, est destinée au dépôt et à la diffusion de documents scientifiques de niveau recherche, publiés ou non, émanant des établissements d'enseignement et de recherche français ou étrangers, des laboratoires publics ou privés. 


\title{
Human-oriented design of collaborative robots
}

\author{
Pauline Maurice $^{\mathrm{a}, *}$, Vincent Padois ${ }^{\mathrm{a}}$, Yvan Measson $^{\mathrm{b}}$, Philippe Bidaud $^{\mathrm{a}, \mathrm{c}}$ \\ ${ }^{a}$ Sorbonne Universités, UPMC Univ Paris 06, CNRS UMR 7222, Institut des Systèmes Intelligents et de Robotique, F-75005, Paris, France \\ ${ }^{b}$ CEA, LIST, Interactive Robotics Laboratory, Gif-sur-Yvette, F-91191, France. \\ ${ }^{c}$ ONERA, 91123 Palaiseau, France.
}

\begin{abstract}
Collaborative robotics is a possible solution to the problem of musculoskeletal disorders (MSDs) in industry, but efficiently designing such robots remains an issue because ergonomic assessment tools are ill-adapted to such devices. This paper presents a generic method for performing detailed ergonomic assessments of co-manipulation activities and its application to the optimal design of collaborative robots. Multiple ergonomic indicators are defined to estimate the different biomechanical demands which occur during manual activities. For any given activity, these indicators are measured through dynamic virtual human simulations, for varying human and robot features. Sensitivity indices are thereby computed to quantify the influence of each parameter of the robot and identify those which should mainly be modified to enhance the ergonomic performance. The sensitivity analysis also allows to extract the indicators which best summarize the overall ergonomic performance of the activity. An evolutionary algorithm is then used to optimize the influential parameters of the robot with respect to the most informative ergonomic indicators, in order to generate an efficient robot design. The whole method is applied to the optimization of a robot morphology for assisting a drilling activity. The performances of the resulting robots confirm the relevance of the proposed approach.
\end{abstract}

Keywords: Digital human model, Dynamic simulation, Collaborative robot design, Sensitivity analysis

\section{Introduction}

Work-related musculoskeletal disorders (MSDs) represent a major health problem in developed countries. They account for the majority of reported occupational diseases and affect almost $50 \%$ of industrial workers [1]. Since MSDs result from strenuous biomechanical solicitations [2], assisting workers with collaborative robots can be a solution when a task is physically demanding yet too complex to be fully automatized (Fig. 1); a collaborative robot enables the joint manipulation of objects with the worker (comanipulation) and thereby provides a variety of benefits, such as strength amplification, inertia masking and guidance via virtual surfaces and paths [3].

In order to design a robot which decreases at best the risk of developing MSDs, an ergonomic assessment of the robot-worker system must be performed throughout the design process. Though standard ergonomic assessments are based on the observation of a worker performing the task [4, 5], digital evaluations now tend to replace physical evaluations in the design process of workstations; digital evaluations - in which a digital human model (DHM) is used to simulate the worker - indeed present several major advantages [6]. Firstly, the simulation enables easy access to detailed biomechanical quantities, which otherwise can only be measured on real humans through complex instrumentation, if at all (e.g. muscle or joint forces). Secondly, different morphologies of workers can easily be tested without the need for a wide variety of real workers. And thirdly, a virtual - instead of a physical - mock-up of the robot is used for digital assessments, thus removing the need to build a new prototype every time a parameter of the robot is tuned. The overall development time and cost is thereby decreased.

${ }^{*}$ Corresponding author: pauline.maurice(at)polytechnique.org

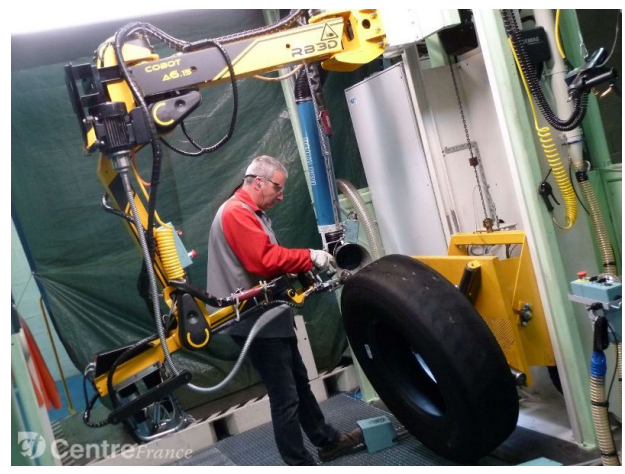

Figure 1: A collaborative robot providing strength amplification for tire retreading (developed by RB3D, CEA-LIST, CETIM).

To perform digital ergonomic evaluations, several commercial DHM software for workplace design provide ergonomic analysis tools (e.g. Delmia, Jack [7], Ramsis [8], Sammie [9]). These software - based on simple rigid-body models of the human body include standard assessment methods which estimate an absolute level of risk depending on the main MSDs factors [2] (posture, effort, duration and frequency of the task) and possibly additional factors (e.g. RULA [10], REBA [11] and OWAS [12] methods, OCRA index [13], NIOSH equation [14]). The resulting ergonomic indicators are, however, either very rough (e.g. effect of external load in RULA) and/or task-specific (e.g. NIOSH equation for lifting loads), so they do not accurately cover all kinds of manual activities which may be addressed by collaborative robots. Besides, these assessment methods are static, i.e. dynamic phenomena are not taken into account; yet fast motions do increase the risk of developing MSDs [15]. In collaborative robotics, evaluating the dynamic phases of 
an activity is even more important because the robot is never perfectly backdrivable and some phenomena cannot be compensated even with a dedicated control law (e.g. additional inertia); manipulating the robot might then require extra efforts and cause new MSDs.

Concurrently to DHM software for workplace design, other DHM software provide more accurate musculoskeletal models of the human body, including muscles, tendons, and bones (e.g. OpenSim [16], AnyBody [17], LifeMOD). Beyond classic macroscopic measurements (joint angles, joint forces and moments), these software also provide dynamic measurements (joint velocities and accelerations) and quantities that more accurately account for the biomechanical demands on the human body (muscle force, tendon deformation, muscle fiber length...). The high number of outputs (one for each muscle/tendon/joint) is, however, difficult to interpret without specific biomechanical knowledge, especially when the purpose is to summarize the global ergonomic level of the activity.

The second criticism which can be addressed to both kinds of DHM software concerns the animation of the DHM. The DHM motion is generated through forward or inverse kinematics, predefined postures and behaviors (e.g. walk towards, reach towards), or from motion capture data. Apart from motion capture, none of these animation techniques enables to come up with a truly realistic human motion. Kinematic techniques do not take into account the inertial properties of the human body or external load, so the simulated motion is rarely human-like [6]. Pre-defined behaviors result in more realistic motions since they rely on a pre-recorded motions database, but only a limited number of behaviors can be simulated and they become unrealistic when external conditions are modified (e.g. adding a load in a reaching motion). In general, the obtained motion is not even dynamically consistent. For instance, the DHM balance is never considered though it affects the relevance of the evaluation [18]. As for motion capture, the human subject and the avatar must experience a similar environment to obtain a realistic simulation. In particular, the interaction forces with the environment are crucial, so the subject must either be provided with a physical mock-up (Fig. 2) or be equipped with complex instrumentation (digital mock-up through virtual reality and force feedback devices). Motion capture is therefore highly time and resource consuming. In order to circumvent the above-mentioned issues, De Magistris et al. developed an optimization-based DHM controller to automatically simulate dynamically consistent motions [19]. The dynamic controller computes DHM joint torques from a combination of anticipatory feedforward and feedback control. It has many advantages over kinematics techniques, such as ensuring DHM balance and generating hand trajectories that are in accordance with some psychophysical principles of voluntary movements. However, though this controller has been successfully used for a virtual ergonomic assessment, the Jacobian-transpose method used in the feedback control does not guarantee the optimality of the solution, because joint torques limits cannot be explicitly included in the optimization.

Eventually, evaluating the ergonomic benefit provided by a collaborative robot requires that the robot be included in the DHM simulation. Though most DHM software can simulate a DHM within a static environment, they cannot simulate the motion of a collaborative robot which depends on its physical interaction with the DHM, both through its control law and through physical interferences.

Thus, despite many available tools for performing virtual er-

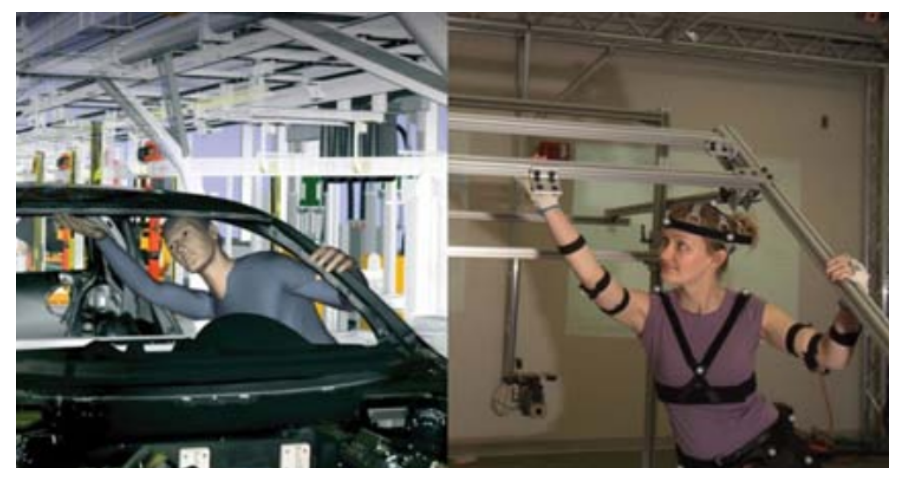

Figure 2: Animation of a DHM using motion capture data, with the Jack software (picture from Jack documentation). The human subject is placed in a physical mock-up of the environment in order to obtain realistic motions.

gonomic assessments, none of them is suitable to evaluate comanipulation activities. This work therefore presents a novel approach for quantitatively comparing the ergonomic benefit provided by different collaborative robots when performing a given activity, and its application to the optimal design of such robots. The proposed method consists in four components (Fig. 3):

1. A list of ergonomic indicators defined to accurately account for the different biomechanical demands which occur during manual activities. They cover all kinds of manual activities, without requiring any a priori hypotheses on the activity that is performed.

2. A dynamic simulation framework in which a DHM can interact with a controlled collaborative robot. The simulation is used to measure the ergonomic indicators. The DHM is animated through an optimization-based whole-body controller to ensure the dynamic consistency of the motion. The controller can be used either with high level tasks descriptions (autonomous DHM, 2a), or with motion capture data (2b). 2a enables the evaluation of robots under development without the need for a human subject or physical mock-ups, while $2 b$ allows the replay of a recorded activity to acquire a reference situation (non-assisted gesture) or evaluate existing robots.

3. A sensitivity analysis framework with which the relevance of each ergonomic indicator and its dependence on the robot parameters can be established - for any given activity - without the need for much input data. The analysis enables the identification of the indicators which best summarize the overall ergonomic performance, and of the robot parameters which most affect this performance. The aforementioned simulation framework is used to automatically create and simulate a variety of situations.

4. A framework for optimizing design parameters of a collaborative robot with respect to relevant ergonomic indicators, based on a multi-objective evolutionary algorithm.

Thanks to the proposed tools, comparing and optimizing the ergonomic benefit provided by collaborative robots is facilitated. The technical gesture is acquired on the initial situation and serves as an input for the sensitivity analysis. The sensitivity analysis enables the identification of a small number of ergonomic indicators relevant for the comparison of robots performances, as well as of the robot parameters to primarily optimize. An optimal robot is then designed with the evolutionary tool by optimizing the relevant ergonomic indicators. The initial (non-assisted) and final (with the 


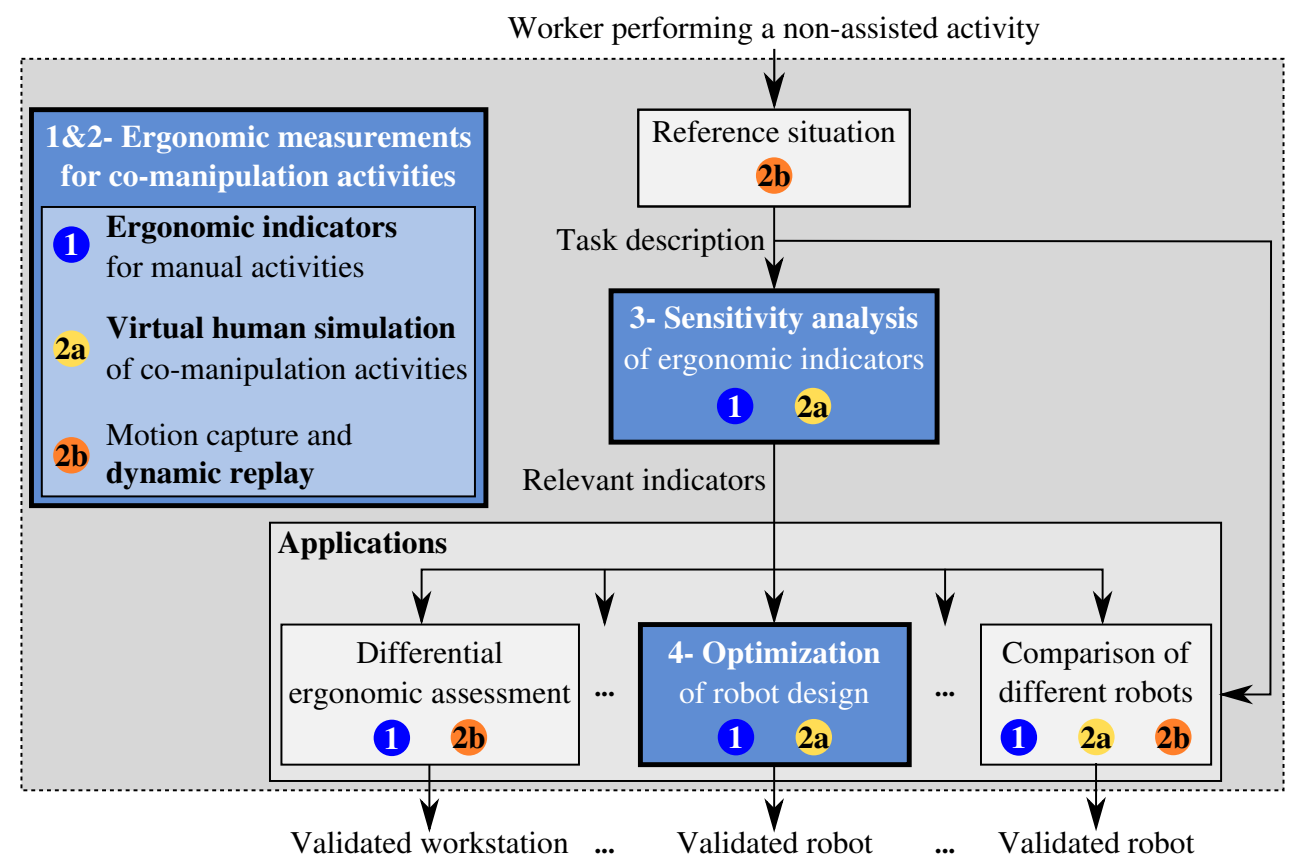

Figure 3: Overview of the methodology developed for performing ergonomic assessments of collaborative robots, and its applications. This paper focuses on the optimization of robot design (4), but other applications are possible.

optimized robot) situations can eventually be compared, to ensure the benefit provided by the robot.

The paper is organized as follows. Section 2 describes the whole methodology (the four components). Section 3 presents an application (protocol and results) of the proposed method, which purpose is the optimization of the morphology of a collaborative robot for a drilling activity. The current limitations of the proposed method are discussed in section 4.

\section{Method}

In digital human modeling, the human body can be represented with different level of detail (rigid bodies, muscles...). The chosen model, however, affects both the biomechanical quantities measured on the model - hence the formulation of ergonomic indicators - and the controller generating the DHM motion (definition of the actuation variables). In this work, the human body is represented with rigid bodies and does not include muscle actuation; each joint is controlled by a single actuator. Even though muscle-related quantities cannot be estimated with such a model, numerous other quantities can be measured to represent the biomechanical demands that occur during whole-body activities (e.g. joint loads, joint dynamics, mechanical energy...). Besides, given the high actuation redundancy of the human musculoskeletal system, computing muscle forces requires to solve the muscle recruitment problem (i.e. which muscles should be activated - among the infinity of possible activation patterns - to perform a given motion). While musculoskeletal models have proved valid and insightful in specific cases, no general criterion has been established yet for the muscle recruitment problem. The realism of the muscle-related measurements can therefore not be ensured in all possible whole-body situations [17, 20, 21, 22]. The questionable gain of information and the significant computational cost then reduces the interest of musculoskeletal models in the current context.

\subsection{Ergonomic indicators for collaborative robotics}

Ergonomic indicators aim at quantifying exhaustively and concisely the physical demands endured by a worker when executing various manual activities, with or without a collaborative robot. Such indicators should take into account the main MSDs risk factors considered in standard ergonomic assessments (posture, force...), but also phenomena that are usually left aside, such as dynamic demands.

In standard ergonomic assessments, risk factors of different nature are often combined together to form a single and compact ergonomic score. Though the combination of several MSDs factors does increase the risk, the way these various factors interact is, however, not well-established in general[4]. The different kinds of demands are therefore represented by separate indicators here, so that the formulation of the indicators is not task-dependent. The proposed ergonomic indicators are classified into two families - constraint oriented indicators and goal oriented indicators - detailed hereafter.

\subsubsection{Constraint oriented indicators}

Constraint oriented indicators are local joint measurements - position, velocity, acceleration, torque and power - which directly represent the relative level of joint demands ${ }^{1}$. For each one of these five quantities, a global indicator $I_{s}$ is obtained for the legs, the right arm, the left arm, and the back (plus head), by summing the squared contributions of every joint in the considered limb (similarly to [24]). Grouping several joints in one indicator decreases the number of indicators - and thereby the complexity of the ergonomic analysis - while accounting for the situation of the whole body (the evaluation cannot be limited to the joints initially affected by MSDs since an ill-adapted robot may relocate the MSDs risk to

\footnotetext{
${ }^{1}$ See [23] for a detailed study and validation of some of these indicators.
} 
other joints). The different limbs of the body can, however, perform very different tasks simultaneously, hence separate indicators for each limb.

The position and torque of each joint are normalized by average physiological limit values before the summing [21, 25]; the capacities of the normalized joints are then all equivalent, rendering the summing more meaningful. For velocity, acceleration, and power, however, joint physiological limits are not well-documented in the literature and the normalization is impossible for now.

\subsubsection{Goal oriented indicators}

Goal oriented indicators are indirect images of the biomechanical demands endured by a worker; they quantify the ability to comfortably perform certain actions (e.g. balance, force exertion). Goal oriented indicators are very compact: one indicator accounts for the whole-body situation.

Balance. Evaluating the balance quality gives an insight into the effort needed to maintain the posture. Unstable balance indeed requires higher muscular effort since the posture must always be corrected to prevent falling. Balance is quantified through two indicators. Balance stability margin represents the capacity to withstand external disturbances; it is evaluated by the sum of the square distances between the Center of Pressure (CoP) and the base of support boundaries [26]. Dynamic balance evaluates the dynamic quality of the balance with the inverse ${ }^{2}$ of the time before the $\mathrm{CoP}$ reaches the base of support boundary, assuming its dynamic remains the same.

Force/Movement generation. The ability to generate forces and movements is evaluated with manipulability measures [27], which are global images of the joint demands needed to perform a motion/force [28]. This work focuses on skilled technical gestures in which the worker knows the trajectories/forces to follow/exert, therefore directional measures are used; the ability to produce endeffector Cartesian force (resp. velocity) in a given direction is evaluated with the inverse ${ }^{2}$ of the hand(s) force (resp. velocity) transmission ratio [29]. The transmission ratio is calculated with the dynamic manipulability [30] to account for the dynamic effects and the non-homogeneity of the human joint capacities.

Vision. Estimating the ability to easily move one's head in various directions gives an insight into the amount of postural change required to follow a visual target (workers tend to look at what they are doing when performing manual activities). The rotational dexterity of the head [27] is therefore used as a vision-related indicator.

Energy. The whole-body kinetic energy is a global measure of human energetic performance, since it is directly associated with the power consumed during an movement [31].

\subsubsection{Main features}

The aforementioned ergonomic indicators are summarized in Table 1 . They are relative indicators, i.e. they enable to quantitatively compare several situations and identify the most demanding one, but they do not assess an absolute level of risk of developing MSDs.

\footnotetext{
${ }^{2}$ So that all ergonomic indicators should be minimized to improve the ergonomic situation.
}

\begin{tabular}{|c|c|}
\hline Indicator definition & Equation \\
\hline $\begin{array}{l}\text { Joint normalized position } \\
\quad(\mathrm{RA}, \mathrm{LA}, \mathrm{B}, \mathrm{L})\end{array}$ & $\frac{1}{N_{j}} \sum_{i=1}^{N_{j}}\left(\frac{q_{i}-q_{i}^{\text {neutral }}}{q_{i}^{\text {max }}-q_{i}^{\text {neutral }}}\right)^{2}$ \\
\hline $\begin{array}{c}\text { Joint normalized torque } \\
(\mathrm{RA}, \mathrm{LA}, \mathrm{B}, \mathrm{L})\end{array}$ & $\frac{1}{N_{j}} \sum_{i=1}^{N_{j}}\left(\frac{\tau_{i}}{\tau_{i}^{\max }(t)}\right)^{2}$ \\
\hline $\begin{array}{l}\text { Joint velocity } \\
\text { (RA, LA, B, L) }\end{array}$ & $\frac{1}{N_{j}} \sum_{i=1}^{N_{j}} \dot{q}_{i}^{2}$ \\
\hline $\begin{array}{c}\text { Joint acceleration } \\
\text { (RA, LA, B, L) }\end{array}$ & $\frac{1}{N_{j}} \sum_{i=1}^{N_{j}} \ddot{q}_{i}^{2}$ \\
\hline $\begin{array}{c}\text { Joint power } \\
(\mathrm{RA}, \mathrm{LA}, \mathrm{B}, \mathrm{L}) \\
\end{array}$ & $\frac{1}{N_{j}} \sum_{i=1}^{N_{j}}\left|\dot{q}_{i} \tau_{i}\right|$ \\
\hline Kinetic energy & $\frac{1}{2} \boldsymbol{v}^{T} M \boldsymbol{v}$ \\
\hline $\begin{array}{l}\text { Velocity transmission ratio } \\
\text { (right hand, left hand) }\end{array}$ & {$\left[\mathbf{u}^{T}\left(J M^{-1} L^{2} M^{-1} J^{T}\right)^{-1} \mathbf{u}\right]^{\frac{1}{2}}$} \\
\hline $\begin{array}{l}\text { Force transmission ratio } \\
\text { (right hand, left hand) }\end{array}$ & {$\left[\mathbf{u}^{T}\left(J M^{-1} L^{-2} M^{-1} J^{T}\right) \mathbf{u}\right]^{\frac{1}{2}}$} \\
\hline Head dexterity & $\frac{\sigma_{\min }}{\sigma_{\max }}$ \\
\hline Balance stability margin & $\frac{1}{N_{b}} \sum_{i=1}^{N_{b}} d_{i}^{2}$ \\
\hline Dynamic balance & $\frac{\left\|\mathbf{v}_{\mathbf{C o P}}\right\|}{d}$ \\
\hline
\end{tabular}

Table 1: Ergonomic indicators for evaluating biomechanical demands in manual activities. $R A$ stands for right arm, $L A$ for left arm, $B$ for back and $L$ for legs. $N_{j}$ is the number of joints in the considered body-part (RA, LA, B or L), $q_{i}$ the angle of joint $i, q_{i}^{\max }$ the joint limit, $q_{i}^{\text {neutral }}$ the neutral joint position, $\dot{q}_{i}$ the joint velocity, $\ddot{q}_{i}$ the joint acceleration, $\tau_{i}$ the joint torque and $\tau_{i}^{\max }$ the joint torque capacity, which decreases with fatigue according to the evolution law proposed by Ma et al. [32]. $v$ is the generalized velocity, $M=M(\mathbf{q})$ the generalized inertia matrix (the dependence on joint configuration $\mathbf{q}$ is dropped in the formulae for the sake of legibility), $J=J(\mathbf{q})$ the Jacobian matrix of the considered end-effector, $\mathbf{u}$ the task direction of interest, and $L=\operatorname{diag}\left(\tau_{i}^{\max }\right)$ contains the joint torque capacities. $\sigma_{\min }$ (resp. $\sigma_{\max }$ ) is the smaller (resp. bigger) singular value of $J_{\text {rot }}^{h} M^{-1} L$, with $J_{r o t}^{h}=J_{\text {rot }}^{h}(\mathbf{q})$ the rotational part of the head Jacobian matrix. $N_{b}$ is the number of base of support boundaries, and $d_{i}$ the distance between the CoP current position and the ith boundary of the base of support. $\mathbf{v}_{\mathbf{C o P}}$ is the $\mathrm{CoP}$ current velocity, and $d$ the distance between the $\mathrm{CoP}$ current position and the base of support boundary along the direction of $\mathbf{v}_{\text {CoP. }}$

Besides, all these indicators are instantaneous quantities, i.e. they can be measured at each moment of the activity. If the time evolution of the indicators may be interesting, the purpose here is to summarize the whole ergonomic situation with only a limited number of values, to facilitate the comparison of the overall ergonomic performance of different collaborative robots. The instantaneous values of each indicator are therefore time-integrated, so that the whole activity is represented with one single scalar value per indicator.

\subsection{Simulation of co-manipulation activities}

In order to numerically evaluate the ergonomic indicators defined in section 2.1, the considered activity must be simulated with a dynamic autonomous DHM, possibly interacting with a collaborative robot. The simulation is run in a dynamic simulation framework based on a physics engine to ensure the physical consistency of the resulting motion and forces. 


\subsubsection{DHM control}

The DHM motion is computed by solving an optimization problem to find the actuation variables (joint torques, accelerations and contact forces) which enable to follow some objectives at best (e.g. hand trajectory, center of mass acceleration), while respecting physical constraints. Unlike analytical control techniques [33, 34], optimization techniques $[35,36,37]$ allow to solve the human kinematic redundancy while explicitly considering both equality and inequality constraints. The biomechanical limits (i.e. joint and actuations limits) are thus guaranteed to be respected. The actuation variables are computed with the linear quadratic programming (LQP) controller framework developed by Salini et al. [38]. The control problem is formulated as follows:

$$
\begin{aligned}
& \underset{\mathbb{X}}{\operatorname{argmin}} \sum_{i} \omega_{i} T_{i}(\mathbb{X}) \\
& \text { s.t. }\left\{\begin{array}{l}
M(\mathbf{q}) \dot{\boldsymbol{v}}+\mathbf{C}(\mathbf{q}, \boldsymbol{v})+\mathbf{g}(\mathbf{q})=S \boldsymbol{\tau}-\sum_{j} J_{c_{j}}^{T}(\mathbf{q}) \mathbf{w}_{\mathbf{c}_{\mathbf{j}}} \\
G \mathbb{X} \leq \mathbf{h}
\end{array}\right.
\end{aligned}
$$

where $\tau$ is the joint torques, $\mathbf{w}_{\mathbf{c}_{\mathbf{j}}}$ the contact wrench of the $j$-th contact point, $\mathbf{q}$ the generalized coordinates of the system, $v$ the generalized velocity concatenating the free-floating base twist and the joint velocities $\dot{\mathbf{q}}$, and $\mathbb{X}=\left(\boldsymbol{\tau}^{T}, \mathbf{w}_{\mathbf{c}}{ }^{T}, \dot{\boldsymbol{v}}^{T}\right)^{T}$. The equality constraint is the equation of motion; $M$ is the inertia matrix of the system, $\mathbf{C}$ the vector of centrifugal and Coriolis forces, $\mathbf{g}$ the vector of gravity forces, $S$ the actuation selection matrix due to the free-floating base, and $J_{c}^{T}$ the Jacobian of contacts. The inequality constraint includes the bounds on the joint positions, velocities, and torques (all formulated in $\boldsymbol{\tau}$ and $\ddot{\mathbf{q}}$ ), and the contact existence conditions for each contact point according to the Coulomb friction model:

$$
\begin{aligned}
& C_{c_{j}} \mathbf{w}_{\mathbf{c}_{\mathbf{j}}} \leq 0 \quad \forall j \\
& J_{c_{j}}(\mathbf{q}) \dot{v}+\dot{J}_{c_{j}}(\boldsymbol{v}, \mathbf{q}) \boldsymbol{v}=0 \quad \forall j
\end{aligned}
$$

where $C_{c_{j}}$ is the linearized friction cone of the $j$-th contact point.

The objective function is a weighted sum of tasks $T_{i}$ (weights $\omega_{i}$ ) representing the squared error between a desired acceleration or wrench and the system acceleration/wrench. The solution is then a compromise between the different tasks, based on their relative importance. The following tasks are defined:

- Operational space acceleration

$$
\begin{aligned}
& \left\|J_{i} \dot{\boldsymbol{v}}+\dot{J}_{i} v-\ddot{\mathbf{X}}_{\mathbf{i}}^{*}\right\|^{2} \\
& \left\|\ddot{\mathbf{q}}-\ddot{\mathbf{q}}^{*}\right\|^{2} \\
& \left\|\mathbf{w}_{\mathbf{i}}-\mathbf{w}_{\mathbf{i}}^{*}\right\|^{2} \\
& \left\|\boldsymbol{\tau}-\tau^{*}\right\|^{2}
\end{aligned}
$$$$
\text { - Joint space acceleration }
$$$$
\text { - Operational space wrench }
$$$$
\text { - Joint torque }
$$

where $\ddot{\mathbf{X}}_{\mathbf{i}}$ is the Cartesian acceleration of body $i$, and $\mathbf{w}_{\mathbf{i}}$ the wrench associated with body $i$. The superscript ${ }^{*}$ refers to the desired acceleration/force. The desired acceleration is defined by a proportional derivative control:

$$
\ddot{\mathbf{z}}^{*}=\ddot{\mathbf{z}}^{\text {goal }}+K_{v}\left(\dot{\mathbf{z}}^{\text {goal }}-\dot{\mathbf{z}}\right)+K_{p}\left(\mathbf{z}^{\text {goal }}-\mathbf{z}\right)
$$

where $\mathbf{z}$ stands for $\mathbf{X}$ or $\mathbf{q}$, and $K_{p}$ and $K_{v}$ are the proportional and derivative gains. The superscript ${ }^{\text {goal }}$ indicates the position, velocity and acceleration wanted for the body or joint (reference trajectory). $\tau^{*}$ and $\mathbf{w}_{\mathbf{i}}{ }^{*}$ are respectively the desired joint torque vector and desired wrench at contact point $i$. They constitute a specification for the tasks to be performed and thus an input for the controller. They must be specified by the user.

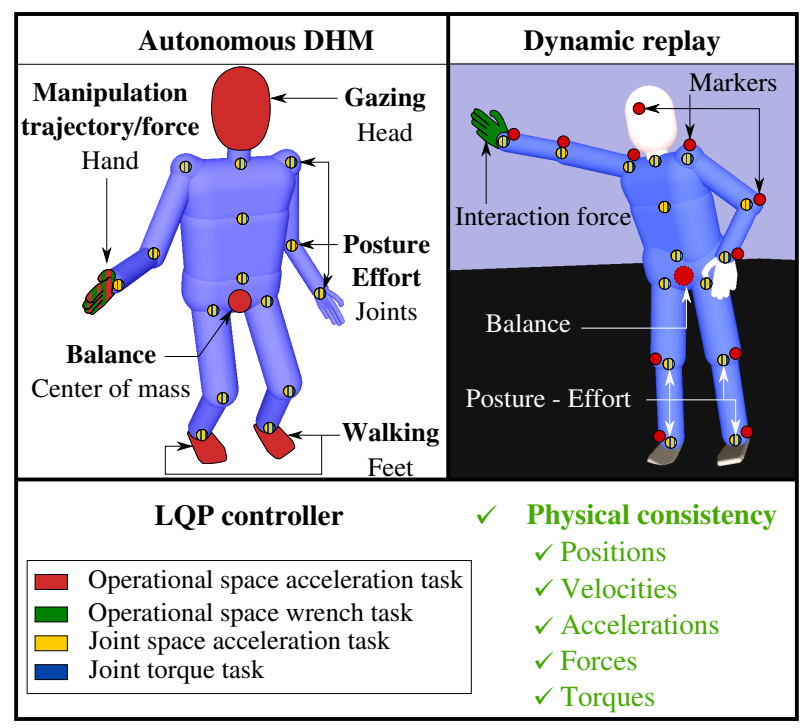

Figure 4: Tasks used in the LQP controller for simulating manual activities with an autonomous DHM (left) or for dynamically replaying human motion (right).

\subsubsection{Animation modes}

The DHM controller presented above is generic and can be used either with motion capture data (replay mode) or with high level tasks descriptions (autonomous mode) (e.g. target to reach, place to go $)^{3}$. In both cases, the DHM balance is managed with a high weight center of mass acceleration task, which reference is computed using a Zero Moment Point preview control [40]. Low weight joint acceleration tasks (postural task) and joint torque tasks are used respectively to define a natural reference posture (standing, arms along the body), and to prevent useless effort.

In autonomous mode, only the body parts that are directly needed to perform the activity - generally one or both hands and the head - are explicitly controlled with an operational acceleration and/or force task (Fig. 4). The reference trajectory for the hand task (manipulation task) results from an interpolation between the start and end points specified by the used. The head is controlled with an orientation task, so that the DHM looks at what it is doing (gazing task). In replay mode, on the contrary, the recorded Cartesian positions of markers positioned on the body of a human subject are mapped onto the DHM. An operational acceleration task is created for each marker, and the reference trajectory is the recorded marker trajectory.

The exact values of the tasks weights are manually tuned through trial and error. Though time consuming in the first place, the tuning process does not need to be repeated; the weights obtained are general enough to be used for successfully simulating many different activities.

\subsubsection{Robot simulation}

This work focuses on collaborative robots which provide strength amplification and are manipulated by the end-effector only (parallel co-manipulation). The simulation method presented in this section is dedicated to such systems specifically.

\footnotetext{
${ }^{3}$ See [39] for a detailed description of the tasks included in the controller in autonomous and replay modes https://hal.archives-ouvertes.fr/ tel-01171482v1/document.
} 
DHM grasp. The DHM fingers are not articulated because grasping requires a complex control of the fingers which is beyond the scope of this work. The human grasp is therefore represented by a 6 DoFs spring-damper system between the DHM palm and the robot end-effector.

Control law. Strength amplification consists in controlling the robot so that the force it exerts on the manipulated tool (or environment) is an amplified image of the force applied by the worker onto the robot ${ }^{4}$. Additionally, the weight of the robot and the viscous friction effects are compensated. The inertial effects, on the contrary, are not compensated because such compensation is hard to implement on real robots due to the difficulty/cost to properly measure joint accelerations. The global strength amplification control law is:

$$
\tau_{\mathbf{r}}=\alpha J_{e e, r}^{T} \mathbf{F}_{\mathbf{v h}}+\mathbf{g}_{\mathbf{r}}\left(\mathbf{q}_{\mathbf{r}}\right)+B \dot{\mathbf{q}}_{\mathbf{r}}
$$

where $\tau_{\mathbf{r}}$ is the vector of robot joint torques, $\mathbf{q}_{\mathbf{r}}$ the robot joint angles, $\dot{\mathbf{q}}_{\mathbf{r}}$ the joint velocities, $\mathbf{g}_{\mathbf{r}}$ the vector of gravity forces, $B$ the matrix of viscous friction coefficients, $J_{e e, r}$ the Jacobian matrix of the robot end-effector, $\mathbf{F}_{\mathbf{v h}}$ the force applied by the DHM on the robot end-effector, and $\alpha$ the amplification coefficient.

\subsection{Sensitivity analysis of the ergonomic performance}

The simulation framework described in section 2.2 enables to measure multiple ergonomic indicators defined in section 2.1. These measurements are, however, not directly useful for the design of collaborative robots. Comparing the overall ergonomic performance of different collaborative robots based on all the ergonomic indicators is indeed not straightforward, because each indicator has a different biomechanical meaning and different indicators may lead to different conclusions. Moreover, the values of the ergonomic indicators per se do not provide any information on how to improve the robot design, i.e. which parameters should mainly be modified to enhance the overall ergonomic performance. To answer these questions, the most informative indicators and their dependence on the robot parameters must be identified. In most cases, however, no straightforward analytical relation between robot parameters and ergonomic indicators can be established. A statistical sensitivity analysis is therefore conducted [41]. This section presents an extended version of the work presented at the IEEE-RAS Humanoids 2014 conference [42].

\subsubsection{Method overview}

Statistical sensitivity analyses rely on the numerical evaluation of the output (ergonomic indicators here) for numerous values of the input parameters, thus requiring a large number of trials. Having a real subject execute the activity in each situation would be too time consuming, therefore the activity is rather simulated with an autonomous DHM. The whole process for analyzing the relevance of ergonomic indicators regarding the comparison of collaborative robots and the influence of the robot parameters can be summarized as follows (Fig. 5):

1. Define the robot parameters which can be altered.

2. Select - among all the possible combinations of parameters values - those that should be tested.

\footnotetext{
${ }^{4}$ In the simulation the interaction force is estimated with the spring-damper system representing the human grasp; on real robots, a force sensor is embedded on the user handle.
}

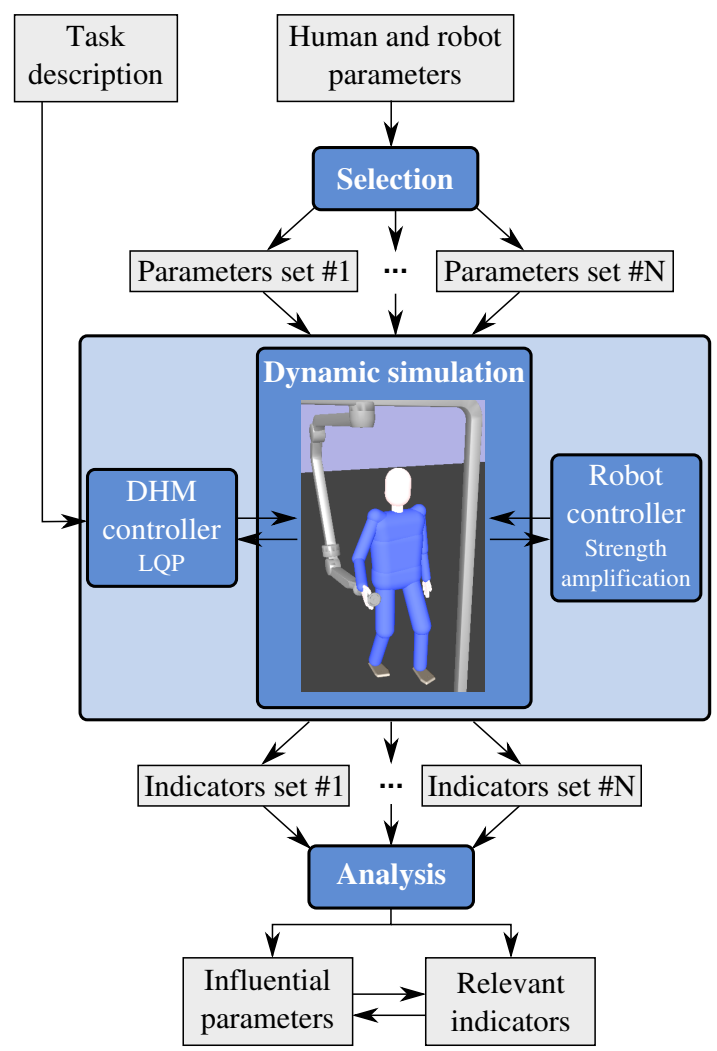

Figure 5: Flow chart of the method for identifying informative ergonomic indicators and influential parameters.

3. Simulate the activity with an autonomous DHM for each selected combination of parameters values, to measure the ergonomic indicators.

4. Compute sensitivity measures for the ergonomic indicators based on their values in all the tested cases.

Steps 1, 2 and 4 are detailed in the following sections. The simulation step 3 is performed with the autonomous DHM as described in section 2.2 .

\subsubsection{Parameters selection}

The sensitivity analysis aims at estimating - for a given activity - how much each parameter of a collaborative robot affects the ergonomic situation. In early stages of a robot design process, however, the number of possible designs - and hence the list of possible parameters - is infinite and there is a priori no reason to choose one over another. In order to be generic, real robot designs are therefore not used. Instead, a robot is modeled by its positive and negative effects on the worker - each effects corresponding to one parameter.

Robot parametrization. In this work, robots are manipulated by the end-effector only (parallel co-manipulation), so the robot is simulated by a 6 DoFs mass-spring-damper system attached to the DHM hand (Fig. 6). The mass $\left(M_{r}\right)$, stiffness $\left(K_{r}\right)$ and damping $\left(B_{r}\right)$ parameters represent the equivalent dynamics of the robot at the endeffector. The possible geometric interferences between the robot and the DHM are simulated without making hypotheses on the robot design, by limiting the DHM movements (limiting the joints range of motion) and modifying its posture (e.g. feet position, joint 


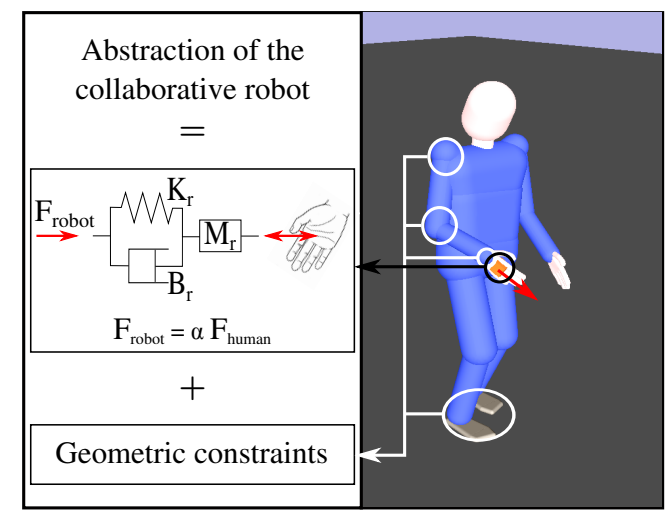

Figure 6: Abstraction of a collaborative robot by a mass-spring-damper system attached to the DHM hand and geometric constraints on the DHM motions (only some examples of constraints are displayed here).

reference position). External forces are applied on the mass-springdamper system to simulate the robot actuation $\left(F_{\text {robot }}\right), \alpha$ being the strength amplification coefficient (Eq. 4). Table 2 gives a concrete example of robot parameters that can be used (these parameters can however be adjusted depending on the activity that is studied).

Parameters space exploration. The robot parameters taking continuous values, they must be discretized to form the different combinations of parameters values to test. But the computational cost of a simulation - though variable - is always expensive (greater or equal than real-time). The number of combinations tested is therefore limited and the values of the parameters must be carefully selected.

Optimizing the exploration of the parameters space requires a compromise between the number of trials and the precision of the resulting information [41]. In this work, the analysis aims at quantitatively estimating the influence of each robot parameter on the ergonomic indicators, to identify which parameters should mainly be tuned. The computation of Sobol indices - which relies on the decomposition of the ergonomic indicators variance (functional ANOVA decomposition) - is then appropriate [43, 44, 45]. Sobol indices allow a fine ranking of the influence of the different parameters, without requiring specific hypotheses on the ergonomic indicators. Furthermore, their interpretation is quite straightforward each index measures the percentage of variance of an indicator that is explained by the corresponding parameter(s). Only the first order indices $S_{i}$ (influence of the parameter $X_{i}$ alone, with no interaction) and the total indices $S_{T_{i}}$ (influence of $X_{i}$, including all interactions with other parameters) are considered in this work, because they give information on the $i$-th parameter independently from other parameters. A high $S_{i}$ means that $X_{i}$ alone strongly affects the indicator, whereas a small $S_{T_{i}}$ means that $X_{i}$ has very little influence on the indicator, even through interactions.

The extended FAST (Fourier amplitude sensitivity testing) spectral method is used for choosing the appropriate parameters values to test (within user-defined bounds) and for computing Sobol indices [46]. The FAST exploration method is indeed a good compromise between the comprehensiveness of the space exploration and the number of trials.

\subsubsection{Ergonomic indicators analysis}

Once the simulations are performed for all the selected combinations of parameters values, Sobol indices can be computed. But
Sobol indices only address single-output models, whereas there are multiple outputs here corresponding to multiple indicators. Even though Sobol indices can be computed separately for each indicator, no global sensitivity index can be obtained for a parameter by aggregating indices relative to different indicators - the comparison of indices referring to different indicators being meaningless. Besides, Sobol indices do not help reducing the number of ergonomic indicators to facilitating the comparison of different collaborative robots. The most informative ergonomic indicators must therefore first be identified.

The purpose of this work is not to assess the absolute level of MSDs risks, but to compare collaborative robots. In this context, the relevance of an indicator is not related to its value, but to its variations when the activity is performed with different robots; if the value of an indicator remains unchanged whichever the robot that is used, this indicator is not useful to compare different robots. The most informative indicators are therefore the ones that best explain the disparity of the results when the activity is performed with various robots.

Ranking. The problem of reducing the number of indicators to keep only the ones that best explain the disparity is addressed by Campbell et al. [47] and Lamboni et al. [48] in the context of sensitivity analysis for multiple-output models. They propose to decompose the model outputs in a well-chosen basis before applying sensitivity analysis to the most informative components individually, which comes down to a dimensionality reduction problem. Standard dimensionality reduction methods, however, cannot be used here, because they form composite variables (i.e. combinations of the initial variables). The ergonomic indicators having different physical meanings, aggregations of various indicators would be meaningless. Moreover, composite variables cannot be used to estimate the global influence of the robot parameters, since the influence of a parameter is likely different from one ergonomic indicator to another. The importance of each ergonomic indicator is therefore represented directly by its variance. The indicators are thus ranked, and the most informative ones (those with the highest variance) are easily identified.

Scaling. Before computing their variance, the indicators must be scaled because they have non-homogeneous units, hence different orders of magnitude. Scaling each indicator with a physiological limit value would be ergonomically meaningful, but some indicators do not have well-defined limits (e.g. kinetic energy), and even the existing ones may be hard to find (e.g. joint acceleration). The order of magnitude (used for the scaling) of an indicator is therefore estimated by measuring the indicator in many different situations with DHM simulations, and taking the average value. Activities of many different kinds (e.g. walking, reaching, pushing, carrying) are performed in many different ways, so the range of values of each indicator is assumed to be covered quite exhaustively ${ }^{5}$.

Selection. Once the ergonomic indicators are ranked according to their variance, a Scree test [49] is performed to decide the number of indicators that are kept; the objective is to limit the number of indicators, while sufficiently accounting for the global ergonomic

${ }^{5}$ The activities used for estimating the indicators order of magnitude are detailed in [42]. A video is available here: http://pages.isir.upmc.fr/ padois/website/fichiers/videos/maurice_humanoids_2014.mp4 
performance of the activity. Sobol indices are then computed separately for each one of the selected indicators. The indices relative to different indicators still cannot be compared, but the overall number of indices is reduced, making the interpretation of the results easier for the user.

\subsection{Evolutionary design of a collaborative robot}

The evaluation framework presented in the previous sections enables to rank robot candidates with respect to their ergonomic performance, and to identify which design parameters are crucial for improving this performance. Designing and modifying test candidates is, however, left to the robot designer, who has to rely on his/her experience (potentially limited since collaborative robotics is a rather new approach) and preliminary studies. This process is both time and resource intensive. To circumvent these problems, optimization techniques are used to guide robots design.

Robots are optimized by coupling an evolutionary algorithm (EA) software [50] with the collaborative robot evaluation framework presented previously. The EA is used for exploring the space of robot designs - i.e. providing robot candidates to evaluate while the simulation tool is used to numerically evaluate the various objectives for each robot candidate (Fig. 7) (here the full robot structure - and not its abstraction - is included in the simulation and interacts with the DHM). EAs are well-suited to address the problem of optimal robot design because they enable optimization over vast and non-continuous search spaces and can handle multiobjectives problems [51]. Optimal collaborative robot design is indeed a multi-objective problem: the robot must be optimized regarding both the task and the worker, and potentially other aspects such as the cost or complexity of the structure. Moreover, these general objectives are often divided into several specific objectives; the worker-oriented objective, for instance, is evaluated through multiple ergonomic indicators.

The EA used here is the Non-dominated Sorting Genetic Algorithm-II (NSGA-II) [52] - based on the Pareto optimality principle - because it efficiently addresses convergence and diversity of the solutions, the two main features that allow to approach the Pareto-optimal front at best. Though NSGA-II (and multi-objective EAs in general) is designed to solve multi-objective problems, the number of objectives affects the convergence of the optimization; the number of conflicting ${ }^{6}$ objectives should generally be limited to three [53]. The ergonomic indicators analysis presented in section 2.3 is therefore used beforehand to select a small number of relevant worker-oriented objectives.

\section{Results}

The whole method for guiding the design of collaborative robots presented in section 2 is applied to a real activity. The motion of a human subject performing the considered activity is recorded and replayed, to evaluate the initial situation as a baseline. Autonomous DHM simulations are then run to perform the sensitivity analysis. The indicators which best summarize the overall ergonomic performance of the considered activity are thereby selected, and the robot parameters which should be tuned to enhance this performance are identified. Optimal values of these parameters - with respect to the

\footnotetext{
${ }^{6}$ Two objectives are conflicting when it is impossible to satisfy both of them simultaneously.
}

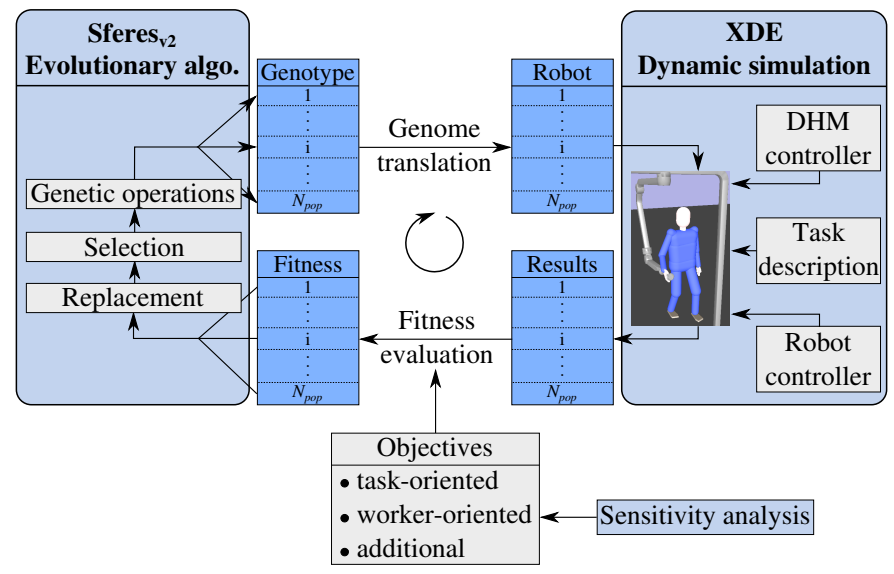

Figure 7: Framework for the optimization of collaborative robot design: XDESferes $_{v 2}$ coupling (a single generation loop is represented). The genetic operations are performed by the $\operatorname{Sferes}_{v 2}$ software [54], which provides a population of robot candidates to evaluate. For each candidate, the objectives are measured through a DHM simulation. To limit the number of objectives, only the most informative ergonomic indicators are included in the worker-oriented objectives.

relevant indicators - are computed using the EA framework. The activity performed with the optimized robot is then compared to the initial (non-assisted) situation.

\subsection{Acquisition of the initial situation}

\subsubsection{Task description}

An industrial manual task requiring significant effort is used as a test case. The activity consists in drilling six holes consecutively in a vertical slab of autoclaved aerated concrete (dimensions: $30 \times 60 \mathrm{~cm}$ ) with a portable electric drill. The locations of the holes are imposed and depicted on Fig. 8. The drill weighs $2.1 \mathrm{~kg}$. The average normal force needed to drill a hole is about $40 \mathrm{~N}$. The task duration is not constrained, but it takes about $1 \mathrm{~min}$ to perform the whole activity (take the drill, drill the six holes, put the drill down). In the experiment, the drill is held with the right hand only. The subject chooses his feet position but is not allowed to move them during the trial.

\subsubsection{Motion capture set-up}

The subject's motion is recorded with a CodaMotion ${ }^{7}$ system at $100 \mathrm{~Hz}$. The subject is equipped with 25 markers spread all over his body. A 6 axes ATI force sensor ${ }^{8}$ is embedded in the drill handle to measure the drilling forces (Fig. 8). The recorded data are filtered with a zero-phase $10 \mathrm{~Hz}$ low pass 4th order Butterworth filter.

\subsubsection{Motion replay}

The motion recorded on the human subject is replayed with a $\mathrm{DHM}^{9}$, using the dynamic replay method described in section 2.2. The simulation is run in the physics-engine based simulation framework XDE developed by CEA-LIST [55]. The XDE DHM consists of 21 rigid bodies linked together by 20 compound joints, for a grand total of 45 degrees of freedom (DoFs) plus 6 DoFs for the

\footnotetext{
${ }^{7}$ www. codamotion. com

${ }^{8}$ ww .ati-ia.com/products/ft/ft_models.aspx?id=Gamma

${ }^{9} \mathrm{~A}$ video is available here: https://www.youtube. com/watch?v= uapVynimoTo
} 


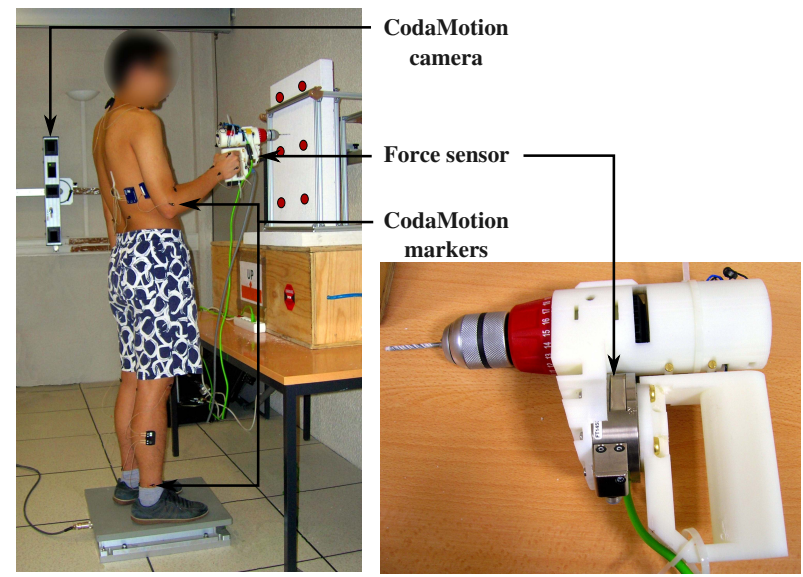

Figure 8: Force and motion capture instrumentation for the drilling activity. A commercial drill has been modified to embed a force sensor. The red circles on the slab represent the drilling points.

free-floating base. Each DoF is a revolute joint controlled by a single actuator. Given a subject's stature and mass, the DHM is automatically scaled according to average anthropometric coefficients $^{10}$, and each body segment is further manually modified to match the subject morphology when needed.

The replayed motion enables the measurements of the ergonomic indicators in the initial situation. But the technical part of the gesture (i.e. the profiles of the tool trajectory and of the drilling force) is also needed to animate the autonomous DHM for the sensitivity analysis simulations ${ }^{11}$.

\subsection{Sensitivity analysis}

\subsubsection{Simulations}

The drilling activity is simulated in the XDE framework, with the autonomous DHM; only the right hand trajectory and force are explicitly specified through a Cartesian acceleration/force task in the DHM controller (plus balance, postural and effort minimization tasks). The hand trajectory and drilling force profile recorded on the human subject serve as reference trajectory/force for the hand tasks. The DHM feet do not move during a simulation (i.e. no automatic stepping), except if the dynamic balance cannot be maintained and the DHM falls. The drill weight is not included in the simulation because it is supported by the collaborative robot. The abstraction of the collaborative robot (section 2.3.2) provides strength amplification during drilling.

\subsubsection{Input parameters}

The input parameters of the sensitivity analysis represent the diversity of potential collaborative robots. In this experiment, only the mass of the robot abstraction (mass-spring-damper system) varies, while the stiffness and damping are kept constant to limit the number of parameters. The geometric interference between the robot and the worker is represented by constraints on the right arm and

\footnotetext{
${ }^{10}$ segments lengths: http://www.openlab.psu.edu/tools/ calculators/proportionalityConstant, segments masses: http://biomech.ftvs.cuni.cz/pbpk/kompendium/biomechanika/ geometrie_hmotnost_vypocet_en

${ }^{11}$ The acquisition of the technical gesture in itself only requires markers on the tool or the subject's hand and the drilling force.
}

\begin{tabular}{|l|c|c|}
\hline Parameter & Min. & Max. \\
\hline DHM stature $(\mathrm{m})$ & 1.65 & 1.80 \\
\hline DHM BMI $\left(\mathrm{kg} . \mathrm{m}^{-2}\right)$ & 21.0 & 27.0 \\
\hline angle pelvis - normal to stab $\left(^{\circ}\right)$ & -30 & 30 \\
\hline offset distance pelvis - center of stab $(\mathrm{m})$ & -0.3 & 0 \\
\hline upper body reference positions $\left(^{\circ}\right)$ & 0,0, & 15,45, \\
& 0,0 & 45,135 \\
\hline upper body joint limits & 0.3 & 1.0 \\
\hline robot mass $(\mathrm{kg})$ & 2 & 10 \\
\hline amplification coefficient & 1 & 3 \\
\hline
\end{tabular}

Table 2: Drilling activity parameters definition and limit values. The pelvis position is given in polar coordinates with respect to the center of the stab. The offset for the pelvis-stab distance is added to the DHM arm length to define the real pelvis-stab distance. The upper-body joint limits are specified as ratio of the regular joint limits and applied to each joint of the back and right arm. The reference positions of the upper-body joints are only modified for the back flexion, shoulder flexion, shoulder abduction, elbow flexion; they are given in the same order and relative to the reference posture (upright, arms along the body). The reference positions of the four joints are not independent to limit the number of parameter.

back (because the robot is manipulated with the right hand) joint limits and joint reference positions, and on the pelvis distance and orientation. The strength amplification coefficient is also included in the parameters. Parameters representing the diversity of workers are added to ensure that the human features do not have a strong impact on the ergonomic situation (otherwise, the robot should include some adjustable parts to adapt to specific workers' morphologies). The worker is defined by his/her stature and body mass index (BMI). The numerical upper and lower bounds of the input parameters are given in Table 2.

The R software sensitivity toolbox ${ }^{12}$ is used to select the parameters values - within the user-defined bounds - that need to be tested for the extended FAST analysis. The sample size and set of frequencies are chosen according to the recommendations of Saltelli et al. [46]. They result in a grand total of 8008 simulations. One simulation takes approximately $2 \mathrm{~min}$ (real time: $75 \mathrm{~s}$ ) on one core of a $2.4 \mathrm{GHz}$ Intel R CoreTM i7 laptop, and the simulations can be parallelized.

\subsubsection{Results}

The 26 ergonomic indicators defined in section 2.1 are analyzed. The velocity and force transmission ratio are computed for the right hand, in the motion direction and in the drilling direction respectively.

Relevant ergonomic indicators. Table 3 presents the five ergonomic indicators - out of 26 in the initial list - that are identified as relevant according to the sensitivity analysis. The five indicators together represent $81 \%$ of the total variance information, therefore only little information is lost by not taking into the other indicators. The selection of the upper-body torque and position indicators is consistent with the physical demands of the drilling activity (exerting a significant force with the right hand while covering a quite extended area). The absence of any velocity or acceleration indicators is consistent with the fact that the drilling activity does not

\footnotetext{
${ }^{12}$ http://www.r-project.org
} 


\begin{tabular}{|c|c|c|c|c|c|c|}
\hline & \multicolumn{5}{|c|}{ Relevant ergonomic indicators } \\
\hline & & $\begin{array}{c}\text { Legs } \\
\text { position }\end{array}$ & $\begin{array}{l}\text { Right Arm } \\
\text { torque }\end{array}$ & $\begin{array}{l}\text { Back } \\
\text { torque }\end{array}$ & $\begin{array}{c}\text { FTR drilling } \\
\text { direction }\end{array}$ & $\begin{array}{l}\text { Right Arm } \\
\text { position }\end{array}$ \\
\hline & & $31 \%$ & $19 \%$ & $14 \%$ & $10 \%$ & $7 \%$ \\
\hline \multirow{16}{*}{ 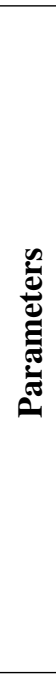 } & \multirow{2}{*}{ DHM stature } & $10^{-3}$ & 0.13 & 0.19 & 0.42 & 0.07 \\
\hline & & 0.03 & 0.15 & 0.29 & 0.52 & 0.12 \\
\hline & \multirow{2}{*}{ DHM BMI } & $10^{-3}$ & 0.05 & 0.02 & 0.21 & $10^{-5}$ \\
\hline & & 0.02 & 0.06 & 0.03 & 0.23 & 0.02 \\
\hline & \multirow{2}{*}{$\begin{array}{c}\text { Pelvis } \\
\text { orientation }\end{array}$} & $10^{-4}$ & 0.10 & 0.01 & 0.15 & 0.15 \\
\hline & & 0.01 & 0.14 & 0.03 & 0.23 & 0.28 \\
\hline & \multirow{2}{*}{ Pelvis distance } & $10^{-3}$ & $10^{-4}$ & 0.01 & 0.02 & 0.03 \\
\hline & & 0.01 & 0.02 & 0.02 & 0.03 & 0.12 \\
\hline & \multirow{2}{*}{$\begin{array}{l}\text { Upper body } \\
\text { ref. position }\end{array}$} & 0.60 & 0.20 & 0.56 & 0.08 & 0.23 \\
\hline & & 0.73 & 0.22 & 0.69 & 0.10 & 0.42 \\
\hline & \multirow{2}{*}{$\begin{array}{l}\text { Upper body } \\
\text { joint limits }\end{array}$} & 0.26 & 0.01 & 0.06 & $10^{-3}$ & 0.28 \\
\hline & & 0.37 & 0.03 & 0.10 & 0.02 & 0.43 \\
\hline & \multirow{2}{*}{ Robot mass } & $10^{-4}$ & $10^{-6}$ & $10^{-5}$ & $10^{-6}$ & $10^{-5}$ \\
\hline & & $10^{-3}$ & $10^{-3}$ & $10^{-3}$ & $10^{-3}$ & 0.02 \\
\hline & \multirow{2}{*}{$\begin{array}{c}\text { Amplification } \\
\text { coefficient }\end{array}$} & $10^{-4}$ & 0.46 & $10^{-5}$ & $10^{-4}$ & $10^{-5}$ \\
\hline & & $10^{-3}$ & 0.49 & $10^{-3}$ & $10^{-3}$ & 0.02 \\
\hline
\end{tabular}

Table 3: Sobol indices for all five ergonomic indicators identified as relevant for the drilling activity. For each parameter and indicator, the upper value is the first order index, the lower value is the total index. The ergonomic indicators are presented in decreasing order of importance (decreasing variance) from left to right: the percentages below their names correspond to the percentage of the total variance they explain. FTR stands for force transmission ratio. Numbers are colored from blue (minimum) to red (maximum), to facilitate the reading.

require fast motions. The presence of the legs joint position indicator as the most discriminating indicator is, however, less expected.

Indicator-Parameter dependence. Some parameter-indicator relations represented by Sobol indices in Table 3 are strongly expected and confirm the consistency of the proposed analysis (e.g. influence of the strength amplification coefficient on the right arm torque indicator, influence of the upper-body geometric parameters on the right arm position indicator). Other relations, however, are less straightforward and could not easily be guessed without the sensitivity analysis (e.g. predominant influence of the upper-body geometric parameters on the legs position indicator, absence of influence of the strength amplification coefficient on the back torque indicator).

Conclusion regarding robot design. The results of the sensitivity analysis highlights two global trends. Firstly, the robot mass does not significantly affect the overall ergonomic performance, since it has no influence on any of the selected indicators. When designing a collaborative robot for the drilling activity, the robot mass is therefore not a critical parameter (from an ergonomic point of view). Secondly, all the selected indicators are significantly affected by at least one of the parameters representing the geometric interference between the robot and the worker. In the drilling activity, the morphology of the robot is therefore critical to the ergonomic benefit provided by the robot.

\subsection{Evolutionary design of a robot morphology}

The efficiency of a collaborative robot being highly taskdependent, designing a robot specifically for a given activity is often preferable to using a generic robot. Designing a dedicated robot from scratch is, however, costly and therefore not accessible to small companies. An intermediate solution is to use a generic platform including modifiable elements.

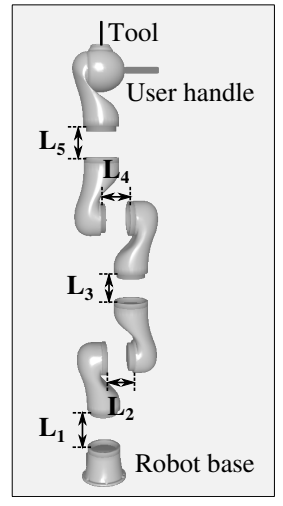

(a) Kuka LWR-like robot.

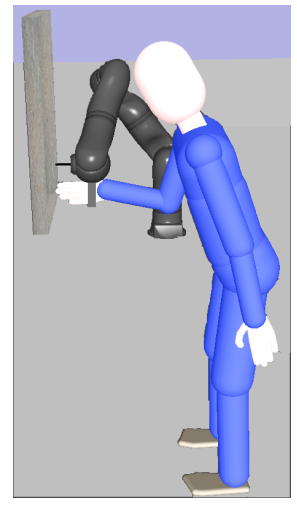

(b) DHM simulation.
Figure 9: DHM simulation of the drilling task, assisted by a 7 DoFs Kuka LWR-like robot with adjustable segments lengths $L_{i}$, and providing strength amplification.

\subsubsection{Optimization variables}

A generic 7 DoFs architecture (similar to a Kuka-LWR) with variable lengths for the first five segments is used (Fig. 9); the robot is manipulated by a user handle mounted on the end-effector. The control law of the robot is not optimized (to limit the complexity of the problem and hence the convergence time), so all robot candidates use the same strength amplification control law with $\alpha=2$ (Eq. 4). The optimization therefore aims at finding optimal values for the segments lengths and for the position and orientation of the robot base ${ }^{13}$.

\footnotetext{
${ }^{13}$ Unlike the sensitivity analysis where an abstraction of the robot is used, the drilling activity is simulated here with the full robots candidates.
} 
Though the physical features of the worker do affect the ergonomic performances (Table 3), only one average human morphology is used in the optimization because the purpose is only to make a proof of concept.

\subsubsection{Objectives}

According to the results of the sensitivity analysis (Table 3), five ergonomic indicators are relevant to assess the drilling task; the optimization should therefore include five worker-oriented objectives (one for each relevant indicator). The right hand FTR is however removed to decrease the number of objectives in the optimization. Indeed, the FTR is mainly affected by parameters which are constant in the present optimization. The right arm and the back torque indicators are gathered into one single indicator, called upper-body torque indicator. Indeed, apart from the strength amplification coefficient which is constant here, the most influential parameters are the same (with similar parameter/indicator trends) for both indicators. A total of three ergonomic indicators (or worker-oriented objectives) are therefore included in the optimization.

The quality of the drilling task execution (task-oriented objective) is evaluated with the maximal position error of the drill extremity during the drilling phases (one objective). No additional objective is used.

Due to the high number of objectives (four), the probability of a robot belonging to the Pareto front is high, except if a very large population size is used. The population size is, however, constrained by the computation time. Having most of the population in the Pareto front - at least early in the optimization - is not desirable because it turns the EA into a random search algorithm. The fitness (objective) values are therefore discretized to limit the number of robots in the Pareto front.

\subsubsection{Evolutionary algorithm parameters}

The population size and the number of generations in the EA result from a compromise between the computation time and the convergence of the solution. A population of 100 individuals and 200 generations are used. One generation is entirely evaluated in about 1 hour on a four-core, $2.4 \mathrm{GHz}$ Intel R CoreTM i7 laptop ${ }^{14}$.

\subsubsection{Results}

The evolution of the four objectives is studied to evaluate the capability of the optimization to find suitable robot morphologies. The optimized robots are then compared with the non-assisted situation to estimate the ergonomic benefit brought by the robot.

Evolution of the objectives. The evolution of the four objectives is displayed in Fig. 10 for the whole population. The mean value of each objective decreases over generations, showing that the overall performance of the robots in the population do improve. Except for the upper-body torque indicator, the objectives minimal values stop evolving almost immediately. The convergence of the objectives maximal values, on the contrary, takes between 100 and 150 generations, especially for the three ergonomic indicators. It is therefore easy to find a robot which performs well on one objective, but finding a robot which matches all four objectives is much harder, hence the usefulness of the optimization.

\footnotetext{
${ }^{14}$ The overall optimization time could however be significantly reduced if a computer with more cores were used because the optimization framework is
} implemented so that several XDE simulations can be run simultaneously.

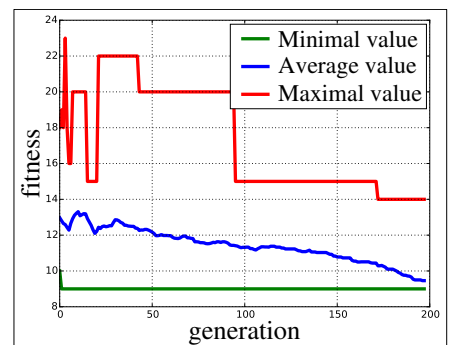

(a) Right arm position indicator

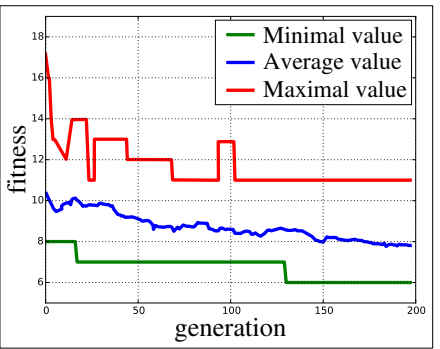

(c) Upper-body torque indicator

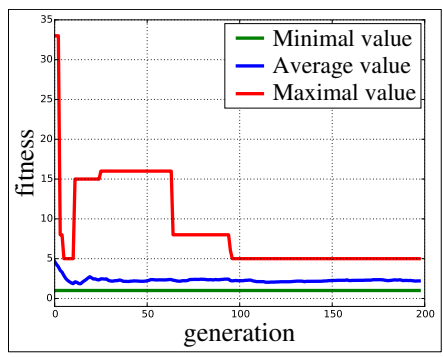

(b) Legs position indicator

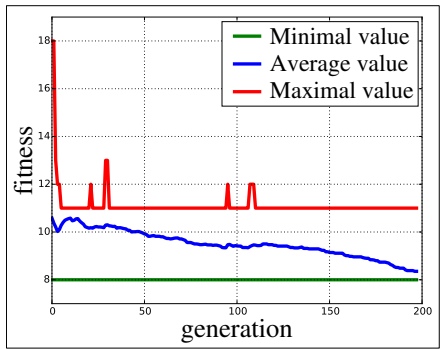

(d) Trajectory error
Figure 10: Evolution of the minimal, average and maximal values of the four objectives over generations (all the robots in the population are included). The discretized fitness values (no units) - and not the ergonomic indicators/trajectory error values - are plotted.

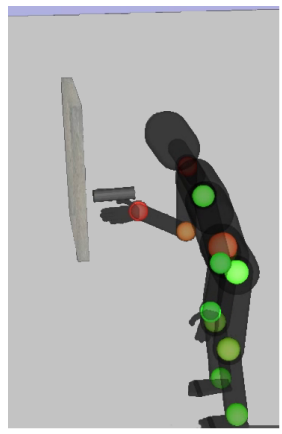

(a) No robot

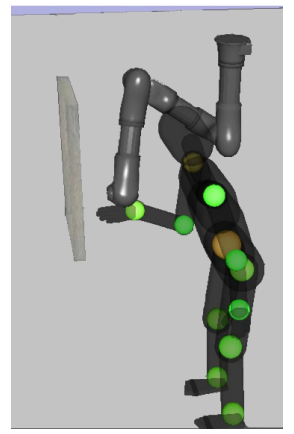

(b) Robot $R_{1}$

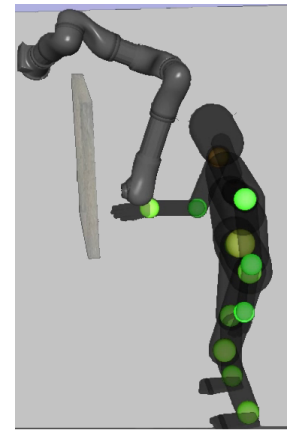

(c) Robot $R_{2}$
Figure 11: Snapshot of the DHM performing the drilling activity without assistance and with the assistance of two near-optimal collaborative robots. The colored spheres represent the instantaneous level of joint effort.

Comparison with the reference situation. During the optimization, the situation with the robot is never compared with the non-assisted situation. Though the robot performances are optimized, there is no certainty that the use of the robot is indeed beneficial. The five ergonomic indicators relevant for the drilling activity (Table 3) are therefore measured in the reference situation (no robot) and with the assistance of two near-optimal robots chosen within the Pareto front of the last generation to represent different solutions (Fig. 11). To make the situations comparable - and as a first validation - all three situations are evaluated with the autonomous DHM (the exact same DHM controller is used $)^{15}$. The results are displayed in Table 4 .

Out of the five relevant indicators, two are significantly improved by both robots (force-related indicators, expected since the robots

\footnotetext{
${ }^{15} \mathrm{~A}$ video of the three simulations is available here https://www. youtube $. \mathrm{com} /$ wat ch $? \mathrm{v}=8 \mathrm{fkT} 6 \mathrm{FSH} 4 \mathrm{e} 0$
} 


\begin{tabular}{|c|c|c|c|}
\cline { 2 - 4 } \multicolumn{1}{c|}{} & No robot & $R_{1}$ & $R_{2}$ \\
\hline Right arm position & 90 & 105 & 125 \\
\hline Legs position & 15 & 25 & 18 \\
\hline Right arm torque & 125 & 38 & 47 \\
\hline Back torque & 75 & 43 & 38 \\
\hline FTR drilling & 130 & 105 & 112 \\
\hline
\end{tabular}

Table 4: Values of the five relevant ergonomic indicators without assistance (No robot) and with the assistance of two near-optimal robots $\left(R_{1}\right.$ and $\left.R_{2}\right)$. For each indicator, the value displayed is the percentage of the indicator reference value (used for the scaling), so that the comparison is more meaningful (the reference value gives an insight into the average order of magnitude of the indicator, however it does not provide any indication on the absolute level of risk). The indicators in red are worsened by the robot, those in green are improved.

provide strength amplification), two remain mostly unchanged, and one is worsened. Despite the degradation in the right arm position indicator, the comparatively significant improvements in the torque and power indicators demonstrate the benefit of the robots. The two near-optimal robots nevertheless show antagonistic performances (e.g. $R_{1}$ is better for the right arm torque but worse for the right arm position), so it is hard to say which one is overall the best (even more when all robots in the Pareto front are considered). The choice between different near-optimal robots is then left to the designer or ergonomist, according to his/her main concerns. The optimization is nevertheless useful, since it performs a pre-selection of the best performing robots. Moreover, the purpose of the optimization is not to replace the designer, but to provide him/her with interesting preliminary designs to be worked on, for further improving the robot performances.

\section{Discussion}

The physically consistent results and the improvement of the robots performances obtained through the optimization demonstrate the usefulness of the proposed method. Its application within the design process of collaborative robots for industrial tasks should, however, be considered carefully because of some current limitations which are discussed thereafter.

\subsection{Limitations of the ergonomic indicators}

Though the ergonomic indicators defined in section 2.1 cover a wide range of MSDs risk factors, the repetitiveness factor is omitted. Yet, repetitiveness belongs to the main MSDs risk factors [2]. The comparison of different collaborative robots is therefore conducted on a single work cycle, and the robot which most decreases the physical demands on one work cycle is assumed to the best overall. But this hypothesis is only valid if the robots do not significantly affect the work rate; this restricts the range of possible applications of the proposed assessment method.

The other time-related risk factor - the duration factor - is taken into account through the time integral value of each ergonomic indicator. This solution comes down to measuring the time spent in different danger zones, each zone being weighed by a danger coefficient equal to the value of the instantaneous demand. But the relation between the time spent in a zone and the risk is very likely not linear. For instance, the same final value can result either from a medium demand all along the task, or from an alternation of strong and light demands. Yet both situations do not have the same biomechanical consequences.
Taking into account the time-frequency aspect of the gesture in the ergonomic evaluation would enable a more accurate assessment, as well as the possibility to extend the possible applications. However, it requires to understand how these time factors affect the human physical capacities, which is closely related to the open problem of fatigue modeling.

\subsection{Limitations of the optimization}

The proposed optimization does improve the performances of the robots, compared to random robots in the initial population. However, the optimization process is useful only if it outperforms the results a robot designer could achieve (i.e. does the optimization provide robots with better or similar performances, in less or comparable time). The answer to this question is not straightforward - neither in general nor for the drilling activity in particular - but several elements must be underlined. Firstly, given the small number of task-related and environment-related constraints and the high number of DoFs of the robot, the drilling activity is not strongly constrained; a good solution would be much less intuitive in a cluttered environment where the optimization may be more useful [56]. The success of the optimization in a cluttered environment, however, strongly depends on the autonomy of the DHM for solving complex problems (e.g. anticipating and avoiding collisions while still reaching the task target); this autonomy is currently limited (see section 4.3). Secondly, the performances of the optimization could be improved by tuning the parameters of the evolutionary algorithm. The values used in this work are based on general recommendations, but no comparative studies have been carried out. The tuning of the parameters should nevertheless not be task-dependent, since the purpose is to provide a generic tool and not to address one specific task.

\subsection{Limitations of the DHM}

Musculoskeletal model. Unlike the muscular actuation of the human body, the actuation of the DHM (in this work) is at joint level only (joint torques), and each DoF is controlled by a single actuator. The biomechanical quantities measured with such a model are therefore less detailed than what could be achieved with a musculoskeletal model. The DHM joint torques, in particular, do not fully represent the overall physical effort exerted by a person. Due to the redundancy of the human actuation, different combinations of muscle forces can result in a same joint torque. Internal muscle forces (i.e. forces which do not generate any joint torque) can thus be generated by a person, but they do not have any equivalent in the DHM model and are therefore not taken into account in the evaluation. Such forces occur during the simultaneous contraction of antagonistic muscles (co-contraction phenomenon) and aim at increasing the joint impedance to withstand perturbations arising from limb dynamics or due to external loads [57]. Though especially important in motions requiring high accuracy, co-contraction occurs in all motions to stabilize the joints and protect joint structures. Not taking co-contraction forces into account therefore leads to an under-estimation of the real human effort. Nevertheless, when comparing several collaborative robots, one can assume that the smaller the effort required to perform the task (not including the cocontraction), the smaller the co-contraction. External efforts (forces to apply on the robot or environment) and gravity-induced efforts (efforts required to maintain a posture) indeed represent a perturbation to the position or force accuracy; if the perturbation is smaller, the stiffness required to resist it is also smaller. A robot which is 
the best regarding the joint torque indicator without considering the co-contraction is therefore likely to be also the best when including co-contraction.

DHM control. Since the sensitivity analysis and the optimization are both based on DHM simulations, the biomechanical reliability of the results strongly depends on the realism of the autonomous DHM motion. The question of feet - as well as other contacts placement is essential, since the activities addressed by collaborative robotics often require significant efforts and thus engage the whole-body. Besides, workers may adapt their feet position during the task, if the robot hinders their gestures. Conversely, the DHM currently lacks autonomy regarding contact placement: the feet positions are entirely set by the user and are therefore not necessarily well-adapted to the task (the DHM can walk or step, but the stepping time and place must be specified beforehand). Solutions for automatic online feet adaptation [58] and for optimal contact placement when significant external forces are at play [59] do exist, but they only partly address the problem. The anticipated (i.e. not purely reactive) optimal placement of contacts indeed requires complex planning methods [60], which for now are too computationally expensive to be used in the current context. More generally, simulating highly realistic human motions requires to understand the psychophysical principles that voluntary movements obey. Many studies have been conducted to establish mathematical formulae of these principles, especially for reaching motions (Fitt's law, minimum jerk principle,...). De Magistris et al. [19] have successfully implemented some of them within the XDE framework, and adding these features in the controller used in this work is a direction for future work. However, these improvements are currently limited to reaching motions because the driving principles are not yet known for all kinds of motions.

Nevertheless, if the results of the sensitivity analysis and optimization presented in this paper are affected by the DHM limitations, the method in itself is independent from the DHM control. Thus in the near future an improved control law could be used to animate the DHM, while the analysis and optimization methods remain the same.

\section{Conclusion}

This paper presents a generic method for performing detailed ergonomic comparisons of collaborative robots and its application to the optimal design of such robots. The whole method is based on DHM simulations and therefore requires only little input data (in particular, no extensive motion capture experiments are needed). For each new activity, ergonomic indicators relevant for robots comparison are automatically selected among about 30 generic indicators, using a sensitivity analysis. Critical design parameters of the robot are identified, and then optimized with an evolutionary algorithm. The whole method is applied to the optimization of a robot morphology to assist a drilling activity. The results of the sensitivity analysis are mostly in accordance with intuitive ergonomic considerations, but they also highlight and quantify less straightforward phenomena. Overall, the enhanced performances of the robots obtained through the optimization demonstrate the usefulness of the proposed approach for easily providing well-performing preliminary robot designs.

Finally, though the framework presented in this work specifically addresses the collaborative robots providing strength amplification, it could easily be adapted for other kinds of collaborative robots, assistive devices, or more generally workstations. Besides, other applications of the sensitivity analysis could be envisaged, such as identifying ergonomically critical phases in complex activities (i.e. phases in which modifications of the robot/workstation have the biggest consequences).

\section{Acknowledgement}

This work was partially supported by the RTE company through the RTE/UPMC chair "Robotics Systems for field intervention in constrained environments", held by V. Padois. The authors would like to thank P. Schlehuber for his significant help for the implementation of the sensitivity analysis and optimization tools .

\section{References}

[1] E. Schneider, X. Irastorza, OSH in figures: Work-related musculoskeletal disorders in the EU - Facts and figures, Tech. Rep., European Agency for Safety and Health at Work, 2010.

[2] A. Luttmann, M. Jäger, B. Griefahn, G. Caffier, F. Liebers, U. Steinberg, Preventing musculoskeletal disorders in the workplace, World Health Organization. Protecting Workers' Health Series 5.

[3] J. Colgate, M. Peshkin, S. Klostermeyer, Intelligent assist devices in industrial applications: a review, Proceedings of the IEEE/RSJ International Conference on Intelligent Robots and Systems (2003) 2516-2521.

[4] G. Li, P. Buckle, Current techniques for assessing physical exposure to work-related musculoskeletal risks, with emphasis on posture-based methods, Ergonomics 42 (5) (1999) 674-695.

[5] G. David, Ergonomic methods for assessing exposure to risk factors for work-related musculoskeletal disorders, Occupational medicine 55 (3) (2005) 190-199.

[6] D. Chaffin, Human motion simulation for vehicle and workplace design, Human Factors and Ergonomics in Manufacturing \& Service Industries 17 (5) (2007) 475-484.

[7] U. Raschke, The Jack human simulation tool, in: Working postures and movements - Tools for evaluation and engineering, CRC Press, 431-437, 2004.

[8] A. Seidl, The RAMSIS and ANTHROPOS Human Simulation Tools, in: Working postures and movements - Tools for evaluation and engineering, CRC Press, 454-462, 2004.

[9] J. Porter, K. Case, R. Marshall, M. Freer, SAMMIE: A Computer-Aided Ergonomics Design Tool, in: Working postures and movements - Tools for evaluation and engineering, CRC Press, 431-437, 2004.

[10] L. McAtamney, E. Corlett, RULA: a survey method for the investigation of work-related upper limb disorders, Applied Ergonomics 24 (2) (1993) 91-99.

[11] S. Hignett, L. McAtamney, Rapid entire body assessment (REBA), Applied Ergonomics 31 (2) (2000) 201-206.

[12] O. Karhu, R. Härkönen, P. Sorvali, P. Vepsäläinen, Observing working postures in industry: Examples of OWAS application, Applied Ergonomics 12 (1) (1981) 13-17.

[13] E. Occhipinti, OCRA: a concise index for the assessment of exposure to repetitive movements of the upper limbs., Ergonomics 41 (9) (1998) 1290-1311.

[14] T. Waters, V. Putz-Anderson, A. Garg, L. Fine, Revised NIOSH equation for the design and evaluation of manual lifting tasks, Ergonomics 36 (7) (1993) 749-776.

[15] W. Marras, S. Lavender, S. Leurgans, S. Rajulu, W. Allread, F. Fathallah, S. Ferguson, The Role of Dynamic Three-Dimensional Trunk Motion in Occupationally-Related Low Back Disorders: The Effects of Workplace Factors, Trunk Position, and Trunk Motion Characteristics on Risk of Injury., Spine 18 (5) (1993) 617-628.

[16] S. Delp, F. Anderson, A. Arnold, P. Loan, A. Habib, C. John, E. Guendelman, D. Thelen, OpenSim: open-source software to create and analyze dynamic simulations of movement, IEEE Transactions on Biomedical Engineering 54 (11) (2007) 1940-1950. 
[17] M. Damsgaard, J. Rasmussen, S. T. Christensen, E. Surma, M. de Zee, Analysis of musculoskeletal systems in the AnyBody Modeling System, Simulation Modelling Practice and Theory 14 (8) (2006) 1100-1111.

[18] D. Lämkull, L. Hanson, R. Örtengren, A comparative study of digital human modelling simulation results and their outcomes in reality: A case study within manual assembly of automobiles, International Journal of Industrial Ergonomics 39 (2) (2009) 428-441.

[19] G. De Magistris, A. Micaelli, P. Evrard, C. Andriot, J. Savin, C. Gaudez, J. Marsot, Dynamic control of DHM for ergonomic assessments, International Journal of Industrial Ergonomics 43 (2) (2013) 170-180.

[20] D. Thelen, F. Anderson, S. Delp, Generating dynamic simulations of movement using computed muscle control, Journal of biomechanics 36 (3) (2003) 321-328.

[21] D. Chaffin, G. Andersson, B. Martin, Occupational biomechanics, Wiley, 4th edn., 2006

[22] J. Hicks, T. Uchida, A. Seth, A. Rajagopal, S. Delp, Is My Model Good Enough? Best Practices for Verification and Validation of Musculoskeletal Models and Simulations of Movement, Journal of biomechanical engineering 137 (2).

[23] P. Maurice, Y. Measson, V. Padois, P. Bidaud, Experimental assessment of the quality of ergonomic indicators for collaborative robotics computed using a digital human model, Proceedings of the 3rd Digital Human Modeling Symposium

[24] C. Pholsiri, Task-based decision making and control of robotic manipulators, Ph.D. thesis, The University of Texas at Austin, 2004.

[25] K. Holzbaur, W. Murray, S. Delp, A model of the upper extremity for simulating musculoskeletal surgery and analyzing neuromuscular control, Annals of biomedical engineering 33 (6) (2005) 829-840.

[26] Y. Xiang, J. Arora, S. Rahmatalla, T. Marler, R. Bhatt, K. Abdel-Malek, Human lifting simulation using a multi-objective optimization approach, Multibody System Dynamics 23 (4) (2010) 431-451.

[27] T. Yoshikawa, Manipulability of robotic mechanisms, The International Journal of Robotics Research 4 (2) (1985) 3-9.

[28] J. Jacquier-Bret, P. Gorce, N. Rezzoug, The manipulability: a new index for quantifying movement capacities of upper extremity, Ergonomics 55 (1) (2012) 69-77.

[29] S. Chiu, Control of redundant manipulators for task compatibility, Proceedings of the IEEE International Conference on Robotics and Automation 4 (1987) 1718-1724.

[30] T. Yoshikawa, Dynamic manipulability of robot manipulators, Proceedings of the IEEE International Conference on Robotics and Automation 2 (1985) 1033-1038.

[31] K. Abdel-Malek, J. Yang, W. Yu, J. Duncan, Human Performance Measures: Mathematics, Tech. Rep., Department of Mechanical Engineering, The University of Iowa, 2005.

[32] L. Ma, W. Zhang, D. Chablat, F. Bennis, F. Guillaume, Multi-objective optimisation method for posture prediction and analysis with consideration of fatigue effect and its application case, Computers \& Industrial Engineering 57 (4) (2009) 1235-1246.

[33] O. Khatib, A unified approach for motion and force control of robot manipulators: The operational space formulation, IEEE Journal of Robotics and Automation 3 (1) (1987) 43-53

[34] L. Sentis, O. Khatib, A whole-body control framework for humanoids operating in human environments, Proceedings of the IEEE International Conference on Robotics and Automation (2006) 2641-2648.

[35] Y. Abe, M. da Silva, J. Popović, Multiobjective control with frictional contacts, Proceedings of the ACM SIGGRAPH/Eurographics symposium on Computer animation (2007) 249-258.

[36] O. Kanoun, F. Lamiraux, P. Wieber, F. Kanehiro, E. Yoshida, J. Laumond, Prioritizing linear equality and inequality systems: application to local motion planning for redundant robots, Proceedings of the IEEE International Conference on Robotics and Automation (2009) 2939-2944.

[37] A. Escande, N. Mansard, P. Wieber, Hierarchical quadratic programming Fast online humanoid-robot motion generation, The International Journal of Robotics Research

[38] J. Salini, V. Padois, P. Bidaud, Synthesis of complex humanoid wholebody behavior: a focus on sequencing and tasks transitions, Proceedings of the IEEE International Conference on Robotics and Automation (2011) 1283-1290.

[39] P. Maurice, Virtual ergonomics for the design of collaborative robots, Ph.D. thesis, Université Pierre et Marie Curie-Paris VI, 2015.
[40] S. Kajita, F. Kanehiro, K. Kaneko, K. Fujiwara, K. Harada, K. Yokoi, H. Hirukawa, Biped walking pattern generation by using preview control of zero-moment point, Proceedings of the IEEE International Conference on Robotics and Automation 2 (2003) 1620-1626.

[41] A. Saltelli, K. Chan, E. Scott, Sensitivity analysis, Wiley, 2000.

[42] P. Maurice, P. Schlehuber, V. Padois, Y. Measson, P. Bidaud, Automatic selection of ergonomie indicators for the design of collaborative robots: A virtual-human in the loop approach, 14th IEEE-RAS International Conference on Humanoid Robots (2014) 801-808.

[43] W. Hoeffding, A class of statistics with asymptotically normal distribution, The annals of mathematical statistics (1948) 293-325.

[44] I. Sobol, Sensitivity estimates for non linear mathematical models, Mathematical Modelling and Computational Experiments (1993) 407-414.

[45] T. Homma, A. Saltelli, Importance measures in global sensitivity analysis of nonlinear models, Reliability Engineering \& System Safety 52 (1) (1996) 1-17.

[46] A. Saltelli, S. Tarantola, K. Chan, A quantitative model-independent method for global sensitivity analysis of model output, Technometrics 41 (1) (1999) 39-56.

[47] K. Campbell, M. McKay, B. Williams, Sensitivity analysis when model outputs are functions, Reliability Engineering \& System Safety 91 (10) (2006) 1468-1472.

[48] M. Lamboni, H. Monod, D. Makowski, Multivariate sensitivity analysis to measure global contribution of input factors in dynamic models, Reliability Engineering \& System Safety 96 (4) (2011) 450-459.

[49] I. Jolliffe, Principal component analysis, Wiley Online Library, 2002.

[50] D. Goldberg, Genetic algorithms in search, optimization, and machine learning, Addison Wesley, 1989.

[51] S. Doncieux, J. Mouret, N. Bredeche, V. Padois, Evolutionary robotics: Exploring new horizons, New Horizons in Evolutionary Robotics (2011) $3-25$.

[52] K. Deb, A. Pratap, S. Agarwal, T. Meyarivan, A fast and elitist multiobjective genetic algorithm: NSGA-II, IEEE Transactions on Evolutionary Computation 6 (2) (2002) 182-197.

[53] K. Deb, Multi-objective optimization using evolutionary algorithms, John Wiley \& Sons, 2001.

[54] J. Mouret, S. Doncieux, et al., Sferesv2: Evolvin'in the multi-core world., Proceedings of the IEEE Congress on Evolutionary Computation

[55] X. Merlhiot, J. Le Garrec, G. Saupin, C. Andriot, The XDE mechanical kernel: Efficient and robust simulation of multibody dynamics with intermittent nonsmooth contacts, Proceedings of the 2nd Joint International Conference on Multibody System Dynamics

[56] S. Rubrecht, E. Singla, V. Padois, P. Bidaud, M. De Broissia, Evolutionary design of a robotic manipulator for a highly constrained environment, in: New Horizons in Evolutionary Robotics, Springer, 109-121, 2011.

[57] P. Gribble, L. Mullin, N. Cothros, A. Mattar, Role of cocontraction in arm movement accuracy, Journal of Neurophysiology 89 (5) (2003) 23962405 .

[58] A. Ibanez, P. Bidaud, V. Padois, Emergence of humanoid walking behaviors from Mixed-Integer Model Predictive Control, Proceedings of the IEEE/RSJ International Conference on Intelligent Robots and Systems (2014) 4014-4021.

[59] M. Liu, A. Micaelli, P. Evrard, A. Escande, Task-driven posture optimization for virtual characters, Proceedings of the 11th ACM SIGGRAPH/Eurographics conference on Computer Animation (2012) 155164

[60] K. Bouyarmane, A. Kheddar, Multi-contact stances planning for multiple agents, IEEE International Conference on Robotics and Automation (2011) 5246-5253. 